\title{
The Role of Location of Traffic Control in Internetwork Gateways
}

\author{
Özgür Ulusoy, Mehmet Baray \\ Department of Computer Engineering and Information Sciences \\ Bilkent University, Ankara, TURKEY
}

This short paper provides the results of an evaluation of window based gateway-to-gateway level congestion control in an interconnected network environment. In [1], an internetwork model is provided and two dynamic control algorithms with different location of internetwork traffic control are proposed. In one of the algorithms, source gateways regulate the traffic flow, while in the other destination gateways provide the control. The algorithms provide further control to static window protocol by adjusting the window size in accordance with the availability of network resources at the destination. Due to lack of space we do not present these algorithms here. Interested readers are referred to [1]

Proposed control algorithms are intended to operate the system below the critical internetwork load that gives rise to congestion at gateways and connected networks. Dynamic control is provided on the basis of estimated load values determined from the measurements of some control variables. Threshold values are specified for the control variables of the algorithms to place an upper bound on the offered in ternetwork load.

In the first algorithm, internetwork window size between network pairs is adjusted by the destination gateway based on the utilization rates of its resources by internetwork messages. In case of a possible congestion, the destination gateway tries to reduce internetwork traffic. If no congestion is observed the message transmission is operated under normal conditions. The control parameters used in sensing a possible congestion are the rejection rate of messages because of full buffer and the utilization of buffers at the destination gateway.

In the second algorithm the control is provided by the gateway next to the source network and applied to each of its destination network independently. When a source gateway senses the congestion at a destination, it limits the number of packets destined to that network by dynamically adjusting its window size. The control parameters used in the algorithm are the retransmission rate and response time of messages transmitted by the source network.

The dynamic window congestion control algorithms have been evaluated as compared to each other as well as static window control protocol. It has been observed that, the dynamic algorithms are effective in preventing throughput degredation and large message delays of internetwork messages.

The comparison of two dynamic algorithms yields the conclusion that, controlling the internetwork messages at destination gateway produces better performance results than the control at the source gateway of messages. This result is due to the fact that, the first algorithm (control at destination gateway) is more effective in restricting the number of messages in transmission for the large values of internet load. In case of a possible congestion, a destination network can easily provide a lack of traffic by throttling all source networks that send messages to it. In the second algorithm each source network tries to prevent congestion at the destinations of their messages by estimating the current load at the destinations.

\section{Reference}

[1] Ö. Ulusoy, M. Baray "Dynamic Window Congestion Control Algorthms in Interconnected Computer Networks", Technical Report CIS-8808, Bilkent University, SERC, 1988. 